\title{
The new event analysis of the Fermi Large Area Telescope
}

\section{Thibaut Desgardin ${ }^{* \dagger}$}

LUPM (Laboratoire Univers et Particule de Montpellier)

E-mail: thibaut.desgardin@univ-montp2.fr

\begin{abstract}
Since its launch on June 11, 2008 the Large Area Telescope (LAT) [1] on-board the Fermi Gamma-ray Telescope ${ }^{\ddagger}$ has been exploring the gamma-ray sky at energies from $20 \mathrm{MeV}$ to over $300 \mathrm{GeV}$. Five years of nearly flawless operations allowed a constant improvement of the detector knowledge and, as a consequence, continuous update of the events selection and the corresponding instrument response parametrization. The final product of this effort is a comprehensive revision of the entire event-level analysis, from the event reconstruction algorithms in each subsystems to the background rejection technique. The potential improvements include a larger acceptance coupled with a significant reduction in background contamination, better angular and energy resolution and an extension of the energy reach below $\sim 100 \mathrm{MeV}$ and in the TeV range. In this paper I will describe the new reconstruction, the event-level analysis, and show the expected instrument performance with emphasis on the low-energy regime $(<100 \mathrm{MeV})$.
\end{abstract}

10th INTEGRAL Workshop: "A Synergistic View of the High Energy Sky" - Integral2014, 15-19 September 2014

Annapolis, MD, USA

\footnotetext{
* Speaker.

$\dagger$ on behalf of the Fermi Large Area Telescope collaboration

†ttp://fermi.gsfc.nasa.gov/
} 


\section{Introduction}

The current LAT event-level analysis was largely developed before launch using Monte Carlo simulations in a series of iterations that we call Passes: Pass 6 was released at launch and followed in August 2011 by Pass 7 [1], which mitigated the impact of some of the limitations of its predecessor. On-orbit experience with the fully integrated detector has revealed some neglected or overlooked issues: primarily the effect of chance coincidences with cosmic rays (that we call Ghost Events). Clear improvements, with the potential to greatly extend the LAT science capabilities, have been identified in all the main areas, including the Monte Carlo simulation of the detector, the event reconstruction and the background rejection.

\section{The Large Area Telescope}

The Fermi Large area telescope has been design to study the gamma ray sky from $20 \mathrm{MeV}$ to over $300 \mathrm{GeV}$. Photons in this energy range can not be focuses and interact mainly via pair production, therefore the detector uses the resulting charged particles to get information of photon direction and energy.To do so, the LAT relies on 3 main subsystems:

- A tracker-converter subsystem (TKR) made of silicon strip detector planes interleaved with tungsten foils to enhance photon interaction probability. There are $18 \mathrm{x}-\mathrm{y}$ detection planes, the first 12 equipped with $0.03 X_{0}$ thin converters (the "front" section, optimized for angular resolution), the following 4 with $0.18 X_{0}$ thick converters (the "back" section, optimized to increase effective area), and the last 2 without converters. The active sensor is a single-sided Silicon Strip Detector with pitch of $228 \mu \mathrm{m}$. In total there are $73 \mathrm{~m}^{2}$ of silicon detectors and $\sim 880 \mathrm{k}$ readout channels, making it the largest silicon tracker operating in space.

- An electromagnetic calorimeter (CAL), is located below the tracker to absorb part of the shower and measure the gamma-ray energy. The CAL is composed of CsI(Tl) crystal bars arranged horizontally in 8 alternating orthogonal layers of 12 crystals each. The crystal dimensions are $27 \times 20 \times 326 \mathrm{~mm}^{3}$, the vertical size is slightly more that one radiation length and the lateral size is slightly less that one Moliere radius. This hodoscopic configuration allows the reconstruction of the longitudinal and lateral development of the shower, and to measure energies up to the $\mathrm{TeV}$ range, despite the modest depth of 8.6 radiation lengths on axis.

- An anti-coincidence detector (ACD), made of plastic scintillator tiles, covers the tracker and provides a veto signal to separate neutral from charged particles.

The LAT design is modular, the building block is a "tower", which includes a TKR module followed by a CAL module. A $4 \times 4$ array of towers are kept together by an aluminium grid (the main mechanical structure). Figure 1 shows how the LAT and its subsystems integrate within the Fermi satellite together with the GBM (Gamma-ray Burst Monitor): the other instrument on-board Fermi. 


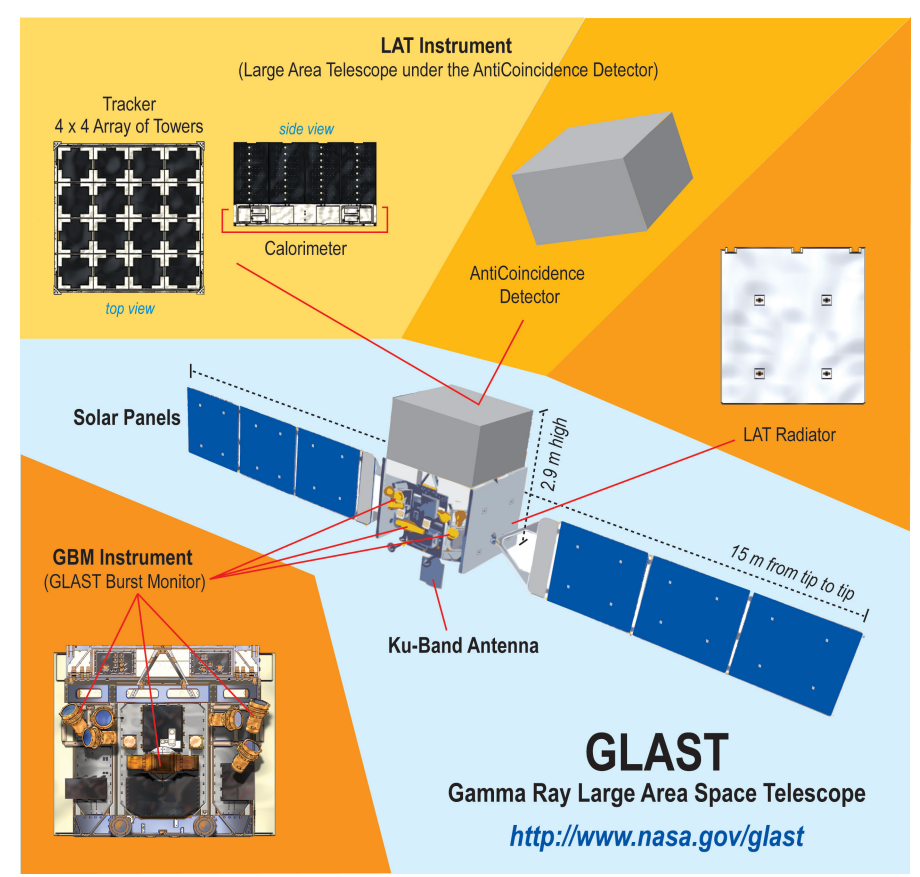

Figure 1: Schematic view of the Fermi satellite featuring the Large Area Telescope (top of the figure) and the Gamma-ray Burst Monitor which is also on-board Fermi. This figure aims to show how the different subsystem of the LAT are integrated on-board the satellite. Source: www.nasa.gov

\section{The new event reconstruction (Pass 8)}

Pass 8 [3], the last iteration of the event analysis, includes a full review of the reconstruction code. These optimizations could lead to extend the LAT science reach especially to the low energy range $(<100 \mathrm{MeV})$, as we will see in section 5. Pass 8 updates the reconstruction algorithm which deals with Ghost events. It was found that Ghost events could cause mistracking and thus decrease the quality of the reconstruction. But Pass 8 also address energy reconstruction in the CAL and background rejection in the ACD as we will see in what follows.

\subsection{Tracker recon}

The Pass 7 tracker reconstruction was based on a track-by-track combinatorics pattern recognition to find the two tacks representing the electron-positron pair.

While it has been very successful in the primary phase of the LAT mission, it has a few limitations. First, the track finding algorithm needs an initial estimate for the particle incident energy and direction. This information is taken from the calorimeter, making the tracker reconstruction intrinsically dependent on the calorimeter one. A second problem is that the current track finding algorithm can be confused by a too large number of hits, created when backsplash particles propagate up from the calorimeter or when photons convert in the back section of the tracker. The consequences of this effect can be the loss of genuine gamma-ray events that fail reconstruction or are mislabelled as background, or the migration of events from the core of the Point-Spread Function (PSF) to the tails because of poorly reconstructed tracks. The Pass 8 reconstruction addresses these issues by introducing a global approach to track finding that tries to model the shower 
development with a tree-like structure. In this process tracker hits are linked together and the tree structure is built by attaching links that share a common hit. The head of the tree represents the assumed gamma-ray conversion point. The tree axis is evaluated with a moment analysis, and is used to associate the tree to a particular cluster in the calorimeter, which allows an estimate of the energy associated with the tree. The first and second tracks are formed with all the hits lying along the two longest, straightest branches of the tree and are fitted with a Kalman Filter technique which accounts for multiple scattering. Tests with Monte Carlo simulations and flight data show that the new tracker pattern recognition has the potential to significantly reduce the fraction of mis-tracked events. As shown in figure 2, most of the Pass 7 misreconstruted events are shifted from the tail to the core of the angular distribution thanks to Pass 8 .

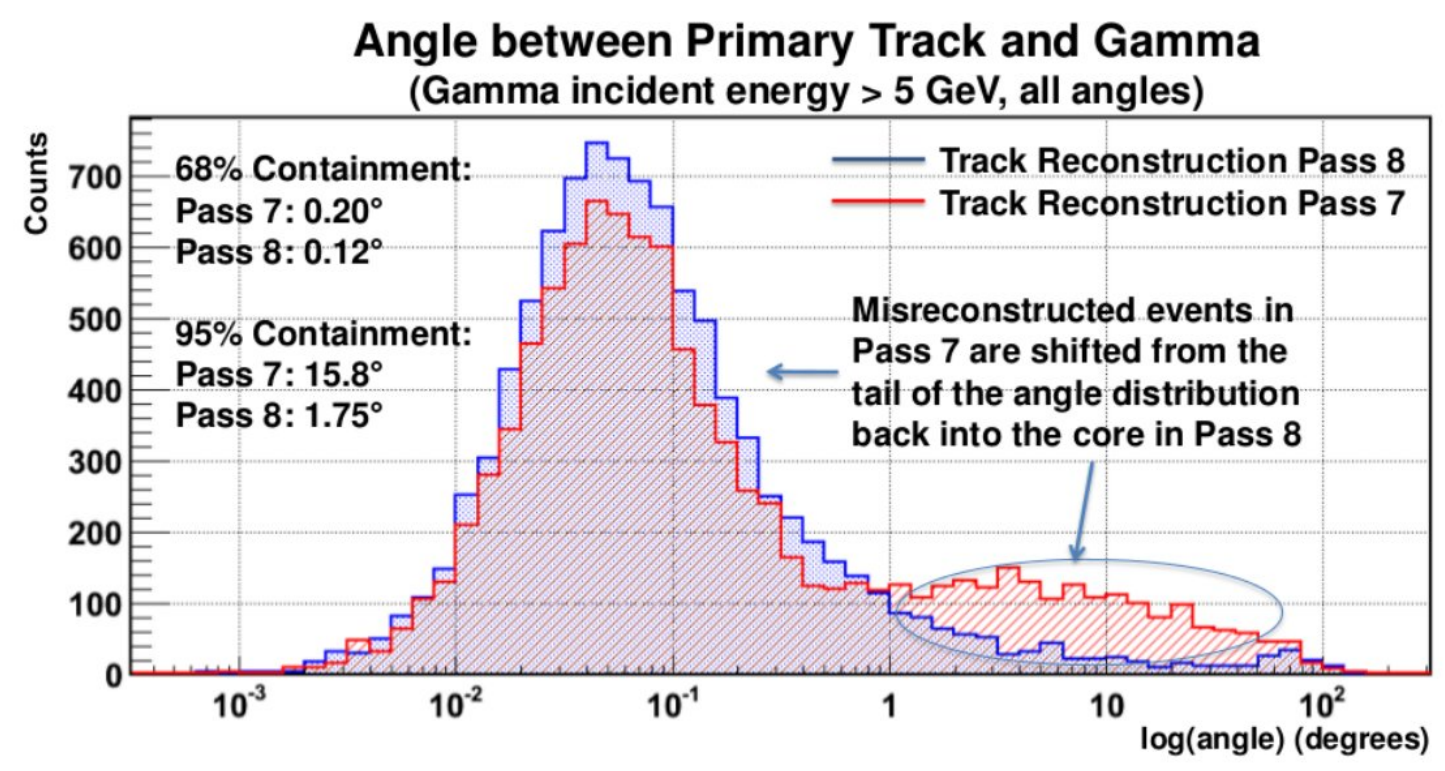

Figure 2: This plot compares the angle between the true and reconstructed direction for the current (Pass 7) with the new (Pass 8) track reconstruction algorithm. The data set is a Monte Carlo simulation of gamma-ray events distributed isotropically with a $1 /$ E energy dependency.

\subsection{Calorimeter reconstruction}

In Pass 7, the CAL reconstruction begins with a shower shape analysis. First the energy centroid is evaluated,then the shower longitudinal axis (that corresponds to the particle direction) is determined through a three-dimensional moment analysis in which the inertia tensor (with energy in place of mass) is diagonalized. The calorimeter direction is very a useful information and its agreement with the TKR direction provides a good discriminant against background contamination. In Pass 8 we abandoned the single-particle paradigm used up to now, and introduced a clustering stage, based on a Minimum Spanning Tree algorithm, with the aim of isolating and removing ghost 
activity. Figure 3 shows an example of a simulated gamma ray, contaminated by ghost energy (taken from LAT periodic triggers and overlayed), and compares the current reconstruction (on the left) with the Pass 8 one that includes the clustering stage (on the right).
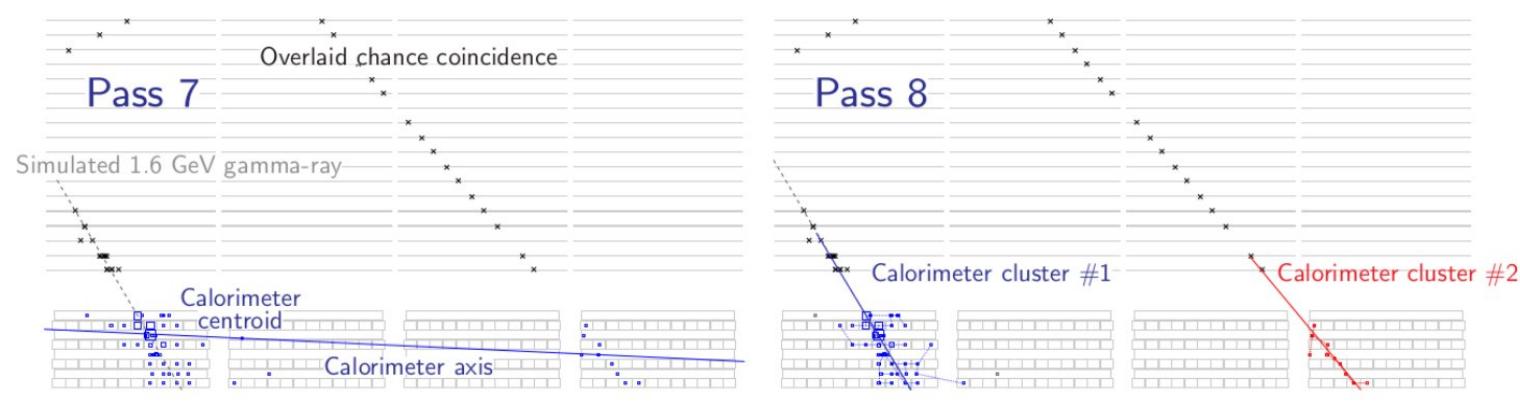

Figure 3: This plot compares the angle between the true and reconstructed direction for the current (Pass 7) with the new (Pass 8) track reconstruction algorithm. The data set is a Monte Carlo simulation of gamma-ray events distributed isotropically with a 1/E energy dependency.

As shown in this event, a small energy deposition far from the main shower can tilt the CAL axis. The result is a poor CAL-TKR matching and the event is discarded. The clustering stage is capable of separating the genuine gamma-ray signal and recovering the event. Moreover it can provide information about the shower topology that can be used in the subsequent stages of the event analysis.

The energy reconstruction is performed with two different algorithms, developed separately for low and high energy regimes. We found that this scheme optimizes the performance in the entire energy range of the LAT. In fact, the CAL absorbs only part of the photon energy, but the energy loss mechanisms are different at low and high energy. As an example, photons below roughly $1 \mathrm{GeV}$ lose a significant fraction of their energy in the TKR; as the photon energy increases, the leakage from the back of the CAL becomes more and more important. To take into account these effects, we implemented a "parametric" method for low-energy photons that uses the total deposited energy and the energy centroid depth along the shower axis, evaluates the energy loss and adds all the contributions together. For higher energies, we found that a full three-dimensional fit of the shower energy deposition is more effective. This method is based on a precise modelling of the longitudinal and lateral development of shower inside the CAL. A reference axis needs to be known and is obtained from the tracker as it provides the best direction resolution. This method works for energies as high as $\sim 1 \mathrm{TeV}$, above which the resolution degrades quickly because of crystal saturation and poor containment. This algorithm has been extensively modified, in the context of the Pass 8 development, in order to improve the handling of saturated crystal in the CAL and extend the energy reach above the $\mathrm{TeV}$ scale. A complete description of the algorithm and its performance can be found in [4]

\subsection{ACD reconstruction}

The ACD reconstruction has been fully re-written. The first major improvement comes from 
the incorporation of calorimeter information when associating incident particle direction with energy deposition in the ACD. Directional information derived from calorimeter clusters is now propagated to the ACD in addition to tracks derived from the tracker. The second major improvement comes from the use of event-by-event direction uncertainties when associating tracks and clusters with energy depositions in the ACD. Previously, the track-tile association was based only on the physical distance and event selection used ad hoc scaling with energy. However, events with the same energy can have different topologies in the tracker and thus large differences in the quality of the reconstructed direction. The last major improvement comes from using the fast ACD signals, provided to the LAT hardware trigger, to mitigate the impact of ghost signals in the slower ACD pulse-height measurements.

\section{Preformances of Pass 8 event classes}

The last step of the Pass 8 development is the high-level analysis that links together all the outputs of the reconstruction and assigns particle properties, such as type, energy and direction. As in previous versions of the event analysis, we use Classification Trees (CTs) to select gamma-ray events and assess the quality of the energy and direction reconstruction. In Pass 8 we use the TMVA [5] multivariate analysis framework and in particular its implementation of Boosted Decision Tree. The CT performance is evaluated from the combination of background rate and gamma-ray acceptance that can be achieved for a given cut on the output signal probability. We have developed event selections for science analysis and used them for the study of possible systematic effects and the assessment of their uncertainties. One of the goals of the Pass 8 effort is in fact to reduce systematic uncertainties in the instrument response function (usually produced via simulations and corrected, if necessary, with flight data). The preliminary Pass 8 acceptance is compared with the current one in figure 4 . We find a $~ 25 \%$ increase at high energies, while at low energy $(<100 \mathrm{MeV})$ the increase can be a factor 2 or more. Monte Carlo predictions, are confirmed by a preliminary analysis of flight data from the Crab pulsar (data points are shown in ratio plot (bottom panel)). 


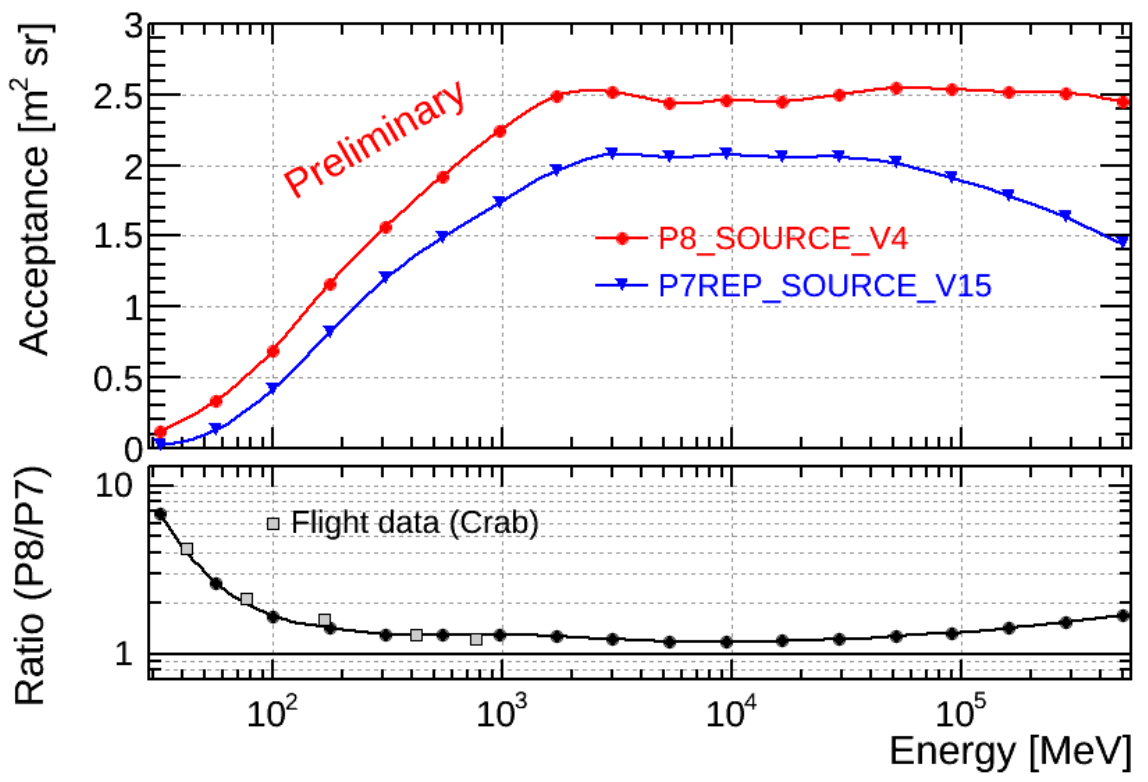

Figure 4: Comparison of new (Pass 8 in red) and current (Pass 7 in blue) performance of the event selection recommended for gamma-ray source analysis. The top plot shows the acceptance as a function of the energy, while the bottom plot shows the ratio Pass $8 /$ Pass 7 . Acceptance ratio, evaluated for preliminary analysis of flight data from the Crab pulsar is shown in ratio plot to confirm Monte Carlo prediction.

The Pass 8 PSF is compared with the current one in figure 5. A clear improvement is visible at high energies where the quality of the reconstruction dominates the angular resolution, while the low-energy behavior is mainly related to multiple scattering. A small caveat is that the Pass 7 PSF contains a correction based on flight data that is not applied to Pass 8 , but preliminary studies suggest that this correction is much smaller with the new reconstruction.

\section{Low Energy Reach}

Additionally to event classes, new event types were also designed. In particular events were classified regarding the quality of their angular reconstruction. They have been classified into 4 types, PSF type 0 contains events within the $0-25 \%$ quantile of a CT that evaluate the quality of the direction reconstruction i.e. events with the worth psf. PSF type 3 contains events with the best psf. Note that the event types cuts do not contain any of the quality or background rejection selections used in the definition of the event classes. Thus, the event type selections should always be used in conjunction with one of the event classes. Figure 6 shows the $68 \%$ containment radius of the PSF, for SOURCE class with different PSF quality types.

Events type were also design with respect to the quality of the energy reconstruction. Together with PSF types, they can be used to mitigate the effect of the broad PSF and energy dispersion at 


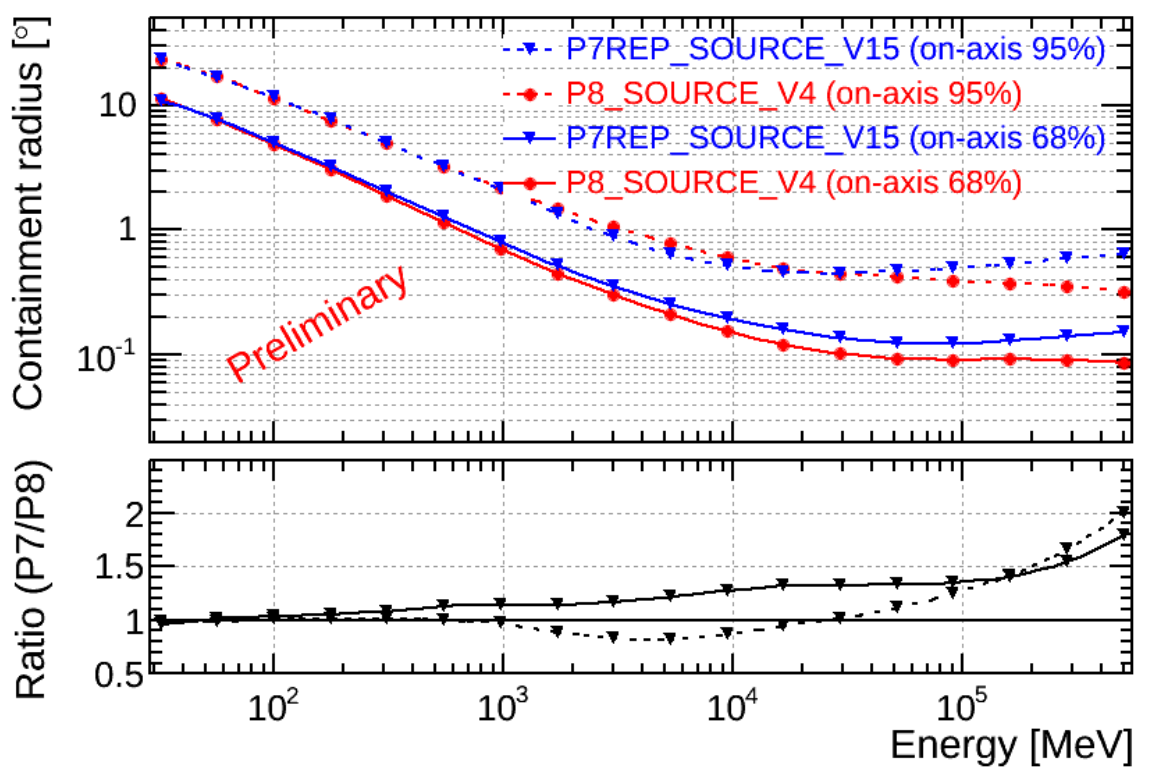

Figure 5: Comparison of new (Pass 8 in red) and current (Pass 7 in blue) PSF 68\% and 95\% containment. The bottom plot shows the ratio to highlight the improvements in particular at high energies. Note that Pass 7 includes an in-flight correction that is not applied to Pass 8, see text for details.

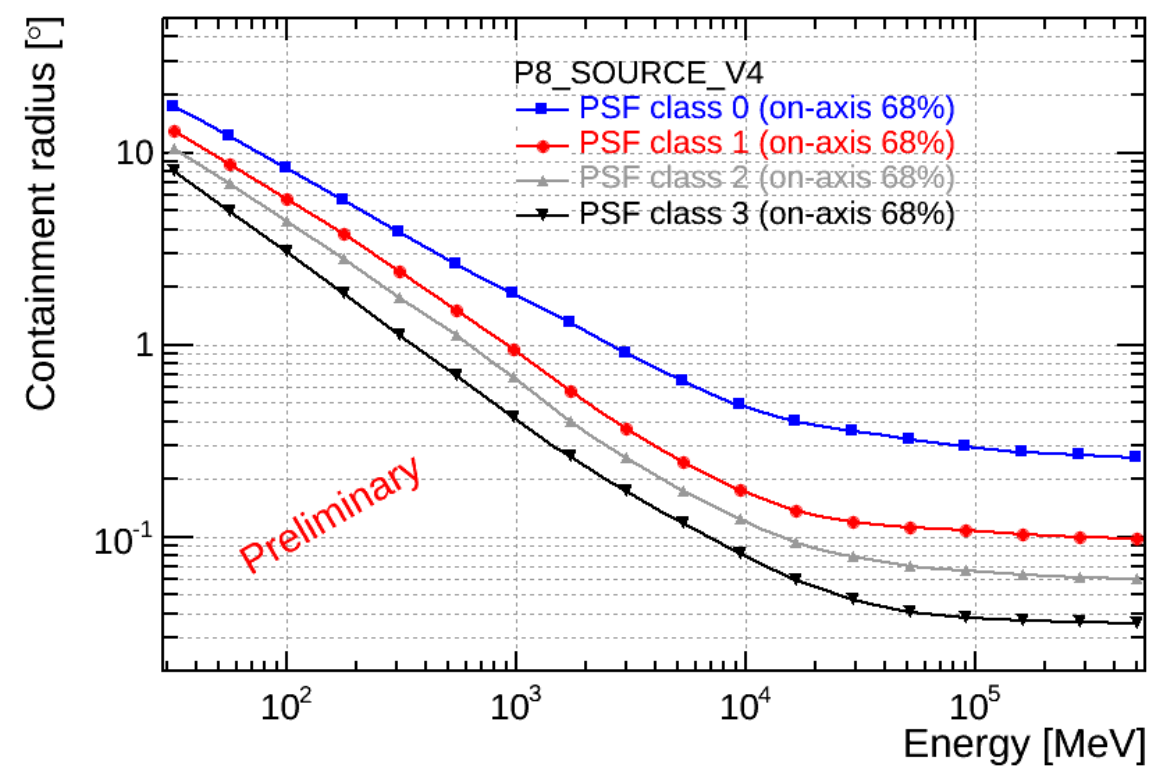

Figure 6: This plot compares the $68 \%$ containment radius of the PSF, for SOURCE class with different PSF quality types. One can see that the $68 \%$ containment radius is $0.4 \mathrm{deg}$ at $1 \mathrm{Gev}$ for PSF type 3 while it is 2 deg for PSF type 0 at the same energy. So it is 5 time smaller with PSF type 3 than PSF type 0 . The data set is a Monte Carlo simulation of gamma-ray events distributed isotropically with a 1/E energy dependency.

low energy $(\mathrm{E}<100 \mathrm{MeV})$. The main features of Pass 8, at low energy are the drastic increase of effective area and the opening of the field view. The higher acceptance will decrease the statistical 
uncertainty at low energies, which will be particularly important for the study of faint objects. Increase in statistics at low energy can be appreciated with the bright Crab pulsar (see figure 7).

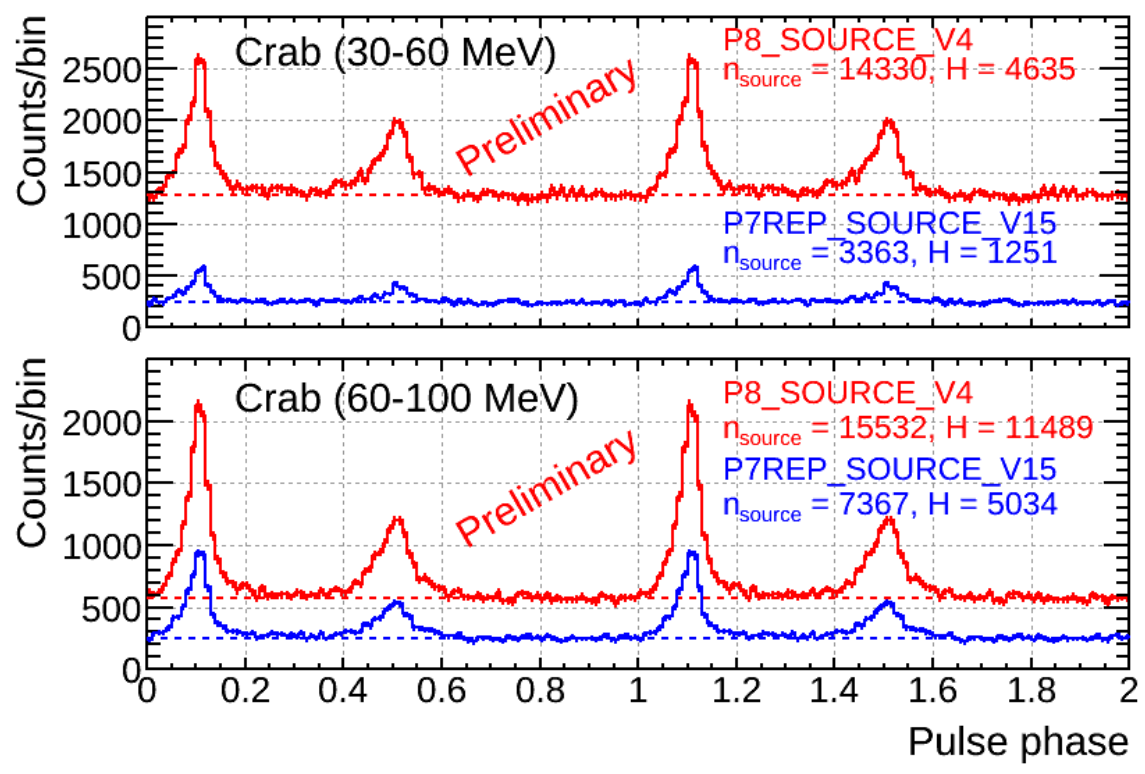

Figure 7: Comparison of new (Pass 8 in red) and current (Pass 7 in blue) background-subtracted pulse profiles for the Crab pulsars (2 years of data, 15 deg ROI) in 2 energy bins below $100 \mathrm{MeV}$.

Low energy science will largely benefit from Pass 8 increase of counts in this range. However particular care on the effect of energy dispersion should be taken for analysis below $100 \mathrm{MeV}$.

\section{Conclusions}

The Fermi-LAT has proven to be an excellent telescope for gamma rays above $\sim 20 \mathrm{MeV}$. All LAT subsystems work as designed with no major failures and no large performance variations. The LAT collaboration is working on a complete upgrade of the event reconstruction and classification pipeline, that will significantly improve the instrument performance and open new scientific opportunities (at low energy for instance). The first step of this process, the new event reconstruction, is complete and frozen. All mission data have been reprocessed with the new reconstruction. The validation and verification of event classes, optimized for different scientific cases of the LAT continues. The Galactic diffuse, the isotropic diffuse and Earth limb models are also checked and will be distributed to the community together with the LAT response functions. Pass 8 has already proven to be an important step forward with its first scientific result already published. Some preliminary results were also shown at Fermi Symposium and will be soon published. The Fermi mission will continue to survey the gamma-ray sky and to provide high-quality data to the whole physics community. 


\section{Acknowledgments}

The Fermi-LAT Collaboration acknowledges support from a number of agencies and institutes for both development and the operation of the LAT as well as scientific data analysis. These include NASA and DOE in the United States, CEA/Irfu and IN2P3/CNRS in France, ASI and INFN in Italy, MEXT, KEK, and JAXA in Japan, and the K. A. Wallenberg Foundation, the Swedish Research Council and the National Space Board in Sweden. Additional support from INAF in Italy and CNES in France for science analysis during the operations phase is also gratefully acknowledged.

\section{References}

[1] Atwood, W. B. et al., The Large Area Telescope on the Fermi Gamma-Ray Space Telescope Mission, The Astrophysical Journal 697(2), 1071 (2009).

[2] Ackermann, M. et al., The Fermi Large Area Telescope On Orbit: Event Classification, Instrument Response Functions, and Calibration, ApJS, 203, 4 (2012) [arXiv:1206.1896]

[3] Atwood, W. B et al., Pass 8: Toward the Full Realization of the Fermi-LAT Scientific Potential, Fermi Symposium 2012 Procs. (2012) [arXiv:1303.3514]

[4] Bruel, P., Gamma rays, electrons and positrons up to $3 \mathrm{TeV}$ with the Fermi Gamma-ray Space Telescope, Journal of Physics: Conference Series 404(1), 012033 (2012).

[5] Hoecker, A., Speckmayer, P., Stelzer, J., Therhaag, J., von Toerne, E., and Voss, H., TMVA: Toolkit for Multivariate Data Analysis, PoS ACAT, 040 (2007). 